\title{
Adaptación lingüística y psicométrica del Cuestionario de Estilos Atributivos Académicos (EAT-A)
}

\author{
Linguistic and psychometric adaptation of the Academic Attributional \\ Style Questionnaire (EAT-A)
}

\author{
Alejandro M. Rodríguez Huitrón ${ }^{\mathrm{a},}$, Laura Hernández-Guzmán ${ }^{\mathrm{a}}$ \\ aLaboratorio de Investigación en Psicopatología Infanto-Juvenil, Facultad de Psicología, \\ Universidad Nacional Autónoma de México, México.
}

\section{Resumen}

Antecedentes: las atribuciones que los estudiantes hacen de sus resultados académicos pueden impactar en su rendimiento académico. Sin embargo, la medición de las atribuciones en este ámbito con frecuencia carece de evidencias de validez y confiabilidad. Objetivos: analizar la estructura factorial, validez convergente y confiabilidad de la adaptación para estudiantes mexicanos del Cuestionario de Estilos Atributivos Académicos (EAT-A). Método: participaron 557 estudiantes de ambos sexos, entre 15 y 19 años, de la Ciudad de México. Mediante el análisis factorial confirmatorio se sometió a prueba una estructura del EATA con siete factores y otra con cuatro que no distingue las atribuciones de éxito y fracaso. Se analizó la validez convergente, la consistencia interna y la confiabilidad compuesta del instrumento. Resultados: solo la estructura con siete factores presentó ajuste adecuado, con los índices RMSEA = .046; SRMR = .053; CFI = .961; TLI = .952 y $\chi^{2} / g \mathrm{l}$ $=2.72$. El EAT-A muestra evidencias de validez convergente con la motivación de logro y de confiabilidad. Conclusión: El EAT-A se muestra como un instrumento de medida breve y fácil aplicación que dispone de evidencias de validez y estimación de confiabilidad para examinar las atribuciones que los estudiantes hacen de sus calificaciones.

Palabras clave: atribuciones causales; calificaciones escolares; educación media superior; instrumentos de medición; análisis factorial.

\section{Para citar este artículo:}

Rodríguez, A. M., \& Hernández-Guzmán, L. (2020).Adaptación lingüística y psicométrica del Cuestionario de Estilos Atributivos Académicos (EAT-A). Liberabit, 26(2), e408. https://doi.org/ 10.24265/liberabit.2020.v26n2.03

\begin{abstract}
Background: Students' attributions of their grades may affect their academic performance. However, lack of evidence supporting the validity and reliability of the instruments measuring attribution is a major concern. Objective: To analyze the factor structure, convergent validity and reliability of the Academic Attributional Style Questionnaire adapted to Mexican high school students (EAT-A). Method: The sample consisted of 557 Mexico City students of both sexes aged between 15 and 19. Using a confirmatory factor analysis for both success and failure attributions, a 7-factor structure and a 4-factor structure of the EAT-A were tested. The instrument's convergent validity, internal consistency and reliability were examined. Results: Only the 7-factor structure showed adequate fit indices: $\mathrm{RMSEA}=.046$; $\mathrm{SRMR}=.053$; $\mathrm{CFI}=.961$; $\mathrm{TLI}=$ .952 and $\chi^{2} / \mathrm{gl}=2.72$. In addition, the EAT-A evidenced convergent validity concerning success and reliability motivation. Conclusion: The EAT-A is a quick- and easyto-administer instrument for measuring students' attributions of their grades in a reliable and valid manner.
\end{abstract}

Keywords: causal attributions; school grading scale; high school; measuring instruments; factor analysis.

Este es un artículo Open Access publicado bajo la licencia Creative Commons Atribución 4.0 Internacional. (CC-BY 4.0)

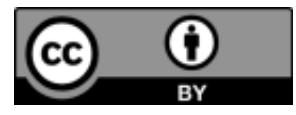

*alejandro8mrh@comunidad.unam.mx, alejandro8mrh@gmail.com
Universidad de San Martín de Porres, Lima - Perú http://ojs3.revistaliberabit.com 


\section{Introducción}

La búsqueda de la causalidad es una actividad que ha favorecido la adaptación del ser humano, ya que permite anticipar los resultados de un evento para determinar un mejor plan de acción. Es así como se intenta saber el porqué de los fenómenos, para entender tanto al ambiente como a nosotros mismos.

Durante su desarrollo, el ser humano asigna una causa, o causas, a un resultado, de tal forma que construye atribuciones que le permiten hacer inferencias para determinar qué ocasiona y mantiene un desenlace (Weiner, 2018). Si bien existe una gran diversidad de posibles causas para un fenómeno, estas se pueden describir en 3 dimensiones: a) locus, si se debe a algo interno o externo de la persona; b) estabilidad, estable versus inestable, por su permanencia en el tiempo y c) controlabilidad, en función del grado en que se considera que uno mismo u otra persona puede modificar esa condición (Graham, 2020; Weiner, 2018). Así, se ha planteado que las atribuciones con las que se explican los resultados de un evento influirán en las expectativas, la motivación, las emociones y el comportamiento de las personas (Pekrun \& Perry, 2014). Al respecto, se ha precisado que el tipo de resultado (positivo o negativo) tiende a atribuirse a diferentes tipos de causas: los resultados positivos, como los éxitos, a atribuciones internas; y los negativos, como los fracasos, a externas (Gonzalez, 2016; Matalinares et al., 2010; Muwonge \& Ssenyonga, 2015; Weiner, 2018).

Uno de los ámbitos en los que frecuentemente se han vinculado las atribuciones es el académico (Houston, 2016; Zubeldia et al., 2018; Sáez, Bustos et al., 2018). La necesidad de examinar las atribuciones en este ambiente es sustancial, ya que la escuela es un espacio de continua evaluación en el que el tipo de atribuciones podrían afectar el potencial de los estudiantes (Dweck \& Yeager, 2019; Farrington et al., 2012; Hamm et al., 2017). Además, las transiciones entre los niveles académicos implican nuevos retos y demandas que podrían favorecer el desarrollo de atribuciones inadecuadas en los estudiantes (Hamm et al., 2016; McMillan, 2015). En el periodo de transición entre la secundaria y la educación superior, dicha situación podría complicarse debido a los cambios cognitivos, físicos y sociales que acompañan la adolescencia (Ahmed et al., 2019; Foulkes \& Blakemore, 2018).

De acuerdo con Weiner (2018), las atribuciones que podrían favorecer los resultados académicos son los siguientes: a) internas, el estudiante es el protagonista del cambio; b) inestables, el éxito o fracaso no es una situación estática, depende en parte de las acciones propias; c) controlables, es posible dirigir determinadas acciones para acercarse al éxito; y d) específicas, las acciones que conllevan al éxito o fracaso son situacionales. Según el autor, estas características permiten que el estudiante considere que tiene el control de los resultados de su aprendizaje.

González-Torres y Tourón (1992) sugieren que lo más importante no es que en determinado momento se realice una atribución, sino que exista una tendencia generalizada por ciertas atribuciones que pueden ser perjudiciales. Estos autores plantean que hay dos patrones atribucionales. Por un lado, los adaptativos, en los que se atribuye los éxitos a la capacidad y al esfuerzo o los fracasos a la falta de esfuerzo. Por otro lado, se encuentran los patrones atribucionales desadaptativos, los cuales se caracterizan por atribuir los éxitos a causas externas e incontrolables, como la suerte, y los fracasos a causas internas, como la capacidad. Bajo esta línea, se ha observado que los patrones adaptativos se asocian con resultados académicos esperados (Gonzalez, 2016; Houston, 2016; Lohbeck et al., 2017).

Además, se ha referido que el tipo de atribuciones influye en la motivación para aprender (Lagos et al., 2016; Pekrun \& Perry, 2014; Sakaki \& Murayama, 2013; Weiner, 2018) y en las metas de logro que se plantean los estudiantes (Matalinares et al., 2010; Miñano \& Castejón, 2011). De acuerdo con Elliot (1999), se distinguen dos metas de logro por las que los estudiantes emprenden las actividades académicas: 
las de dominio y las de competencia. Las metas de dominio conllevan el esfuerzo por aprender o comprender información y desarrollar o perfeccionar una habilidad. Las metas de competencia dirigen la conducta a demostrar buenos resultados ante una evaluación, sin importar si se aprende o mejora en esa actividad (Elliot, 1999). Asimismo, pueden presentar un perfil de acercamiento cuando se realizan las tareas con el mínimo esfuerzo que se requiere solo por cumplir con ellas, o de evitación, el cual consiste en alejarse de las actividades que puedan resultar en un fracaso. Aunque ambas metas de competencia tienen el mismo objetivo, demostrar habilidad, el tipo de aproximación puede afectar la concentración y la persistencia para alcanzar los resultados académicos (Regueiro et al., 2018). En esta línea, se ha observado que las atribuciones internas y controlables se asocian con mayor motivación para realizar tareas académicas (Erten \& Burden, 2014) y metas de dominio que las atribuciones externas, estables e incontrolables (Haynes et al., 2009; Matalinares et al., 2010; Miñano \& Castejón, 2011). Asimismo, se ha planteado, mediante intervenciones, que las atribuciones son maleables y que promover en los estudiantes atribuciones adaptativas conlleva a mejoras en sus resultados académicos (Hamm et al., 2017; Haynes et al., 2009; Mahmoodi \& Doosti, 2018). Por tanto, las atribuciones académicas se vislumbran como una alternativa viable y eficaz para mejorar los resultados académicos de los estudiantes.

Desafortunadamente, existe un problema a la hora de medir las atribuciones en el ámbito académico, el estado de desarrollo incipiente o cuestionable de los instrumentos empleados para tal fin. Por ejemplo, algunos estudios emplean preguntas aisladas para referirse a las atribuciones, sin analizar sus propiedades psicométricas (Carpi et al., 2009); otros estudios se limitan a informar la consistencia interna del instrumento de medida (mediante el alfa de Cronbach), pero no ofrecen mayor evidencia del modelo de medida con el que se está evaluando (Redondo et al., 2014). Asimismo, se observa que comúnmente se recurre de manera errónea al análisis de componentes principales como un procedimiento de análisis factorial para examinar la estructura factorial del modelo de medida de los instrumentos (Alavi et al., 2020). Sin embargo, obviar o no disponer de evidencias de validez y confiabilidad de los instrumentos de medición impide realizar conjeturas válidas de los resultados obtenidos. Al respecto, Clark y Watson (2019) advierten que la evidencia de validez y confiabilidad de un instrumento no es permanente, por lo que es recomendable corroborar las propiedades de los instrumentos cada vez que se aplican.

Como se observa, la medición de las atribuciones en el ámbito académico presenta ciertas limitaciones. Por ejemplo, a pesar de que el Cuestionario de Estilos Atribucionales (EAT) (Alonso \& Sánchez, 1992) carece de evidencia empírica que corrobore su estructura factorial, ha sido uno los instrumentos más empleados debido a su delimitación y definición teórica y a los análisis psicométricos de los ítems, especialmente con población hispanohablante. Este ha sido utilizado con estudiantes de secundaria chilenos (Moraga, 2017), españoles (Miñano \& Castejón, 2011), argentinos (Gómez et al., 2011) y peruanos (Matalinares et al., 2010; Matalinares et al., 2009; Jaimes, 2015). Es por ello que resulta necesario y relevante aportar evidencia empírica que sustente el modelo de medida que se propone en el EAT para examinar las atribuciones que los estudiantes hacen de sus calificaciones.

El EAT es un autoinforme con 72 ítems de respuesta tipo Likert con cinco posibilidades de respuesta $(0=$ totalmente en desacuerdo a $4=$ totalmente de acuerdo). Este instrumento se divide en EAT-Académico (EAT-A), relativo a los estilos atributivos relacionados con los resultados académicos y EAT-Interpersonal (EAT-I), que se refiere a los éxitos y fracasos en las relaciones interpersonales. Sin embargo, debido al interés en las atribuciones académicas, en este estudio solo se hará referencia al EAT-A. Esta escala está compuesta por 41 ítems englobados en siete factores que exploran las causas más comunes a las cuales los estudiantes atribuyen 
el éxito y el fracaso académicos. Jurado et al., (2016) proponen una estructura de cuatro factores en la que, contrario a lo que se indica en la literatura (Matalinares et al., 2010; Muwonge \& Ssenyonga1, 2015; Weiner, 2018), no se diferencian las atribuciones de éxito y fracaso.

En virtud a lo mencionado, así como a la relevancia que tienen las atribuciones en los resultados académicos, el objetivo de este estudio es examinar si la estructura factorial de EAT-A corresponde a su modelo de medida y presenta validez convergente, así como confiabilidad en población mexicana para indagar el tipo de atribuciones que los estudiantes de educación media superior hacen de sus calificaciones. Disponer de mayor certeza del modelo de medida que propone el instrumento permitirá hacer inferencias adecuadas sobre las atribuciones. Esta información permitirá dirigir intervenciones orientadas al desarrollo de atribuciones en los estudiantes que beneficien su formación académica (Hamm et al., 2017; Morris, 2013; Sukariyah \& Assaad, 2015).

\section{Método}

La presente investigación es un estudio instrumental (Montero \& León, 2007) para la revisión de la estructura factorial, validez convergente y confiabilidad del EAT-A en estudiantes mexicanos de bachillerato.

\section{Participantes}

Se realizó un muestreo no probabilístico por conveniencia (Otzen \& Manterola, 2017) de tres instituciones de la zona metropolitana de la Ciudad de México (dos públicas y una privada), así como del grupo de participantes, el cual estuvo conformado por 599 estudiantes de educación secundaria alta (media superior - bachillerato), de acuerdo con la Clasificación Internacional Normalizada de la Educación (UNESCO, 2011). Los criterios de inclusión fueron los siguientes: a) cursar la educación media superior, b) ser estudiante regular, c) firmar el consentimiento informado, d) no presentar alguna limitación para contestar los instrumentos. El criterio de exclusión consistió en no completar los instrumentos. Se prescindió de 42 casos con más de $40 \%$ de omisiones. La muestra final fue de 557 participantes, 458 de escuelas públicas y 99 de privadas; de los cuales 127 eran de primer semestre, 99 de tercero y 331 de quinto. De estos, 285 eran mujeres y 272 hombres con edades que oscilaban entre los 15 y 19 años $(M=16.9 ; D E=.93)$.

\section{Instrumentos}

Cuestionario de estilos atributivos (EAT) de Alonso y Sánchez (1992). El EAT es un autoinforme con 72 ítems de respuesta tipo Likert con cinco posibilidades de respuesta $(0=$ totalmente en desacuerdo a $4=$ totalmente de acuerdo). Si bien este instrumento hace referencia a las atribuciones académicas (EAT-A) y a las atribuciones interpersonales (EAT-I), por la relevancia que posee para este estudio, solo se analizará el EAT-A. Dicha escala está compuesta por 41 ítems distribuidos en siete factores: 1) atribución del éxito al esfuerzo (e. g., Cuando he trabajado con empeño, generalmente he podido superar los obstáculos que me impedían tener éxito en los estudios); 2) atribución del fracaso a la falta de esfuerzo (e. g., Si las notas que saco no son tan buenas como esperaba, normalmente pienso que se debe a mi falta de esfuerzo); 3) atribución del éxito a la habilidad (e. g., Pienso que mis buenas notas reflejan, sobre todo, lo listo que soy para los estudios); 4) atribución del fracaso a la falta de habilidad (e. g., Si sacase malas notas, dudaría de mi inteligencia); 5) atribución del fracaso al profesor (e. g., Normalmente, si he sacado malas notas ha sido porque el profesor era un tacaño al puntuar); 6) atribución del éxito a la suerte (e. g., Por lo general, apruebo simplemente por suerte); $y$ 7) atribución del fracaso a la suerte (e. g., Me parece que, normalmente, mis notas bajas se han debido a la casualidad). Los autores refieren que los siete factores del EAT-A explican el $44.99 \%$ de la varianza, mientras que el coeficiente alfa de Cronbach de cada una de las siete escalas oscila entre .65 y .78. Por su parte, Matalinares et al. (2009), informaron que con estudiantes peruanos 
cada uno de los ítems presenta una $\mathrm{V}$ de Aiken igual o superior a $.88(p<.05)$ y el instrumento completo, un alfa de Cronbach de .62. Con población mexicana, Jurado et al. (2016) examinaron la estructura factorial de una adaptación del instrumento en estudiantes universitarios y encontraron una estructura de cuatro factores: habilidad, esfuerzo, suerte y profesor.

Escala de patrones adaptativos para el aprendizaje ( Patterns of Adaptive Learning Survey», PALS) (Midgley et al., 2000). La PALS es un instrumento de autoinforme que explora, según la Teoría de Orientación a la Meta, los propósitos o razones por las cuales el estudiante se involucra y persiste en situaciones académicas (Elliot, 1999; Regueiro et al., 2018). Emplea ítems tipo Likert con cinco posibilidades de respuesta $(1=$ totalmente falso a 5 = totalmente verdadero). Contiene 106 ítems distribuidos en cinco subescalas, una respecto a las metas académicas que tienen los estudiantes y cuatro con relación a la percepción del estudiante sobre las metas académicas que tienen sus profesores, sus compañeros, sus padres y la comunidad respecto a él. En este estudio se utilizará la subescala que explora las metas académicas de los estudiantes, la cual se denomina patrones individuales de Orientación a la Meta (PALS-PI), que está integrada por 13 ítems que componen tres factores: 1 ) orientación a metas de dominio (OMD) (e. g., Es importante para mí aprender mucho este año); 2) orientación a metas de competencia-acercamiento (OMCA) (e. g., Una de mis metas es demostrar a los otros que soy bueno en el trabajo de clases); y 3) orientación a metas de competencia-evitación (OMCE) (e. g., Una de mis metas es evitar que los demás piensen que no soy listo). Una calificación alta en cada factor representa el tipo de orientación que el estudiante refiere. Los autores del instrumento (Midgley et al., 2000) indican los siguientes coeficientes de confiabilidad (alfa de Cronbach) para cada uno de los factores: OMD = .76 , OMCA $=.70$ y $\mathrm{OMCE}=.83$. En población mexicana y española se informan coeficientes (alfa de Cronbach) para cada escala de .75, .66 y .71, respectivamente, así como una estructura factorial consistente con el instrumento original (Gaeta, Teruel, \& Orejudo, 2012). En esta muestra se obtuvieron los siguientes coeficientes omega de Mcdonald: .81, .78 y .80, respectivamente; así como un buen ajuste de la estructura de tres factores $\left(\chi^{2} / \mathrm{gl}=1.95\right.$; RMSEA $=.034$; IC 90\% [.023, .045]; CFI = .98; TLI = .97; SRMR $=.034)$.

\section{Procedimiento}

En cuanto a la adaptación lingüística del EAT-A, de acuerdo con Muñiz et al. (2013), tres expertos en el área de evaluación educativa, revisaron el contenido del cuestionario para determinar si el lenguaje empleado era adecuado para población mexicana. Asimismo, un grupo de cinco estudiantes de quinto año de bachillerato revisaron los ítems para indicar si algo no les quedaba claro. Posteriormente, se elaboró una versión final con 41 ítems que consideraban las observaciones de los expertos y de los estudiantes. En la versión final, se sustituyeron términos del español de España por palabras que se usan en México y se despersonalizaron los ítems para evitar confusión al responder (e. g., «Si suspendo una asignatura, probablemente es por no estar capacitado intelectualmente para la misma» frente a «Reprobar una materia significa que no se está capacitado intelectualmente para la misma»).

Con el consentimiento de las autoridades correspondientes, se llevó a cabo la aplicación del EAT-A y la PALS-PI de manera colectiva en el salón de clase de cada grupo escolar. Se comentó a los estudiantes el propósito del estudio, así como las características de su participación: voluntaria, anónima y autónoma. Se solicitó a los participantes que revisaran y firmaran el consentimiento informado. Se explicó y ejemplificó la manera de responder y se solicitó que antes de contestar leyeran las instrucciones y ejemplos del cuadernillo de preguntas. El tiempo de aplicación fue de 30 a 45 minutos. 


\section{Análisis de datos}

Se eliminaron los casos que presentaron más de $40 \%$ de omisiones en las respuestas de las escalas (Rikoon et al., 2015). Los casos con un menor porcentaje de omisiones se asignaron mediante el método de imputación múltiple (Lang \& Little, 2018) con el paquete MICE (Buuren et al., 2019) del programa estadístico RStudio versión 1.1.456 (RStudio, 2019). Con el paquete Lavaan (Rosseel et al., 2020), del mismo programa, se realizó el análisis factorial confirmatorio para someter a prueba la estructura factorial del EAT-A. Se utilizó el método de mínimos cuadrados ponderados robusto (WLSMV, por sus siglas en inglés) para la estimación de los parámetros debido a que los ítems del EAT-A son de tipo Likert con 5 opciones de respuesta (MartínezAbad \& Rodríguez-Conde, 2017; Monroe, 2018). De acuerdo con la recomendación de Orcan (2018), cuando se emplea el análisis factorial confirmatorio es necesario no solo corroborar el ajuste de un modelo teórico, sino que además es recomendable comparar los índices de ajuste de otros modelos alternativos para seleccionar el mejor. Dado lo anterior, se sometieron a prueba dos modelos. Por un lado, se puso a prueba el modelo original con la estructura factorial propuesta por el autor, con siete factores; y por otro, un modelo alternativo propuesto por Jurado et al. (2016), con una estructura de cuatro factores: 1) atribución a la suerte, 2) atribución al esfuerzo, 3) atribución a la habilidad y 4) atribución al profesor. Se analizó el ajuste de cada modelo y se eliminaron los ítems con carga menor de .50 en cada factor (Hair et al., 2014).

Para evaluar el ajuste de los modelos se emplearon los siguientes índices: el error cuadrático medio de aproximación (RMSEA) y el residual cuadrático estandarizado (SRMR) como medidas absolutas de ajuste; el índice de ajuste comparativo (CFI) y el índice Tucker-Lewis (TLI) como medidas de ajuste incremental; así como la razón de Chicuadrado sobre los grados de libertad $\left(\chi^{2} / \mathrm{gl}\right)$ como medida de ajuste de parsimonia. De acuerdo con los estándares, los valores adecuados son RMSEA $\leq .06$, SRMR $\leq .06$, CFI $\geq .95$, TLI $\geq .90$ y $\chi^{2} / \mathrm{gl} \leq 3(\mathrm{Hu}$ $\&$ Bentler, 1999; Kline, 1998).

Con el programa RStudio versión 1.1.456 (RStudio, 2019) y el paquete MVN (Korkmaz et al., 2019), se realizó la prueba de Mardia para examinar la normalidad bivariante entre los factores de los instrumentos (Cerrón, 2016). Con el paquete Psych (Revelle, 2019), se exploró la correlación del puntaje de las escalas con las puntuaciones referidas de motivación de logro para determinar la validez convergente del instrumento. Al respecto, Campbell y Fiske (1959) sugieren que existe evidencia de validez convergente cuando los factores de un mismo constructo correlacionan altamente entre ellos y que esa correlación es mayor que la que existe con respecto a las medidas propuestas para otro constructo. Con el mismo paquete se realizó un análisis del coeficiente de omega de Mcdonald para determinar la confiabilidad en términos de consistencia interna de los factores del modelo con mejor ajuste (Deng \& Chan, 2017). De acuerdo con Ventura-León y Caycho-Rodríguez (2017), un coeficiente omega igual o superior a .65 refiere adecuada confiabilidad del instrumento. Asimismo, por tratarse de un análisis factorial confirmatorio, se calculó la confiabilidad compuesta de los factores (Peterson \& Kim, 2013). 


\section{Resultados}

La Tabla 1 muestra el número de factores, ítems e índices de ajuste del modelo original, con siete factores, y del modelo alternativo, con cuatro. En ambos casos es posible apreciar que los indicadores muestran que no hay un ajuste adecuado del modelo con los datos. Dado lo anterior, se eliminaron en ambos modelos los ítems que no discriminaban con sesgo o con carga menor de .50 en cada factor (Hair et al., 2014). De dicho procedimiento resultó una versión B del modelo original, integrada por 23 ítems y una versión B del modelo alternativo, compuesta por 24 ítems. Los índices que muestran un mejor ajuste corresponden a la versión B del modelo original, en el cual se observa distinción entre las atribuciones que los estudiantes hacen del éxito y las del fracaso.

En la Tabla 2 se observan las cargas factoriales de los ítems en cada factor, así como las correlaciones entre factores del modelo de siete factores con 23 ítems.

En cuanto a la validez convergente, con la prueba de Mardia se encontró que los factores de los instrumentos no muestran normalidad bivariante entre ellos, por lo que la asociación entre los factores se examinó con la correlación rho de Spearman. En la Tabla 3 se presentan las correlaciones entre los factores del EAT-A con los patrones de orientación a meta que explora el PALS-PI. En general, se observa que aunque existe asociación entre los factores del EAT-A y del PALS-PI, esta asociación es menor que la existente entre las escalas del EAT-A.

Los factores del modelo original con 23 ítems obtenidos en el análisis factorial confirmatorio alcanzan coeficientes omega Mcdonald y confiabilidad compuesta igual o superior a .65 (Tabla 4), lo que indica una consistencia interna de las escalas adecuadas (Ventura-León \& Caycho-Rodríguez, 2017).

Tabla 1

Índices de ajuste del modelo original y alternativo

\begin{tabular}{lcccccccc}
\hline \multicolumn{1}{c}{ Modelo } & Factores & Ítems & RMSEA & Rango RMSEA & SRMR & CFI & TLI & $\chi^{2} / \mathrm{gl}$ \\
\hline Original & 7 & 41 & .065 & $.065-.068$ & .089 & .858 & .846 & 3.33 \\
Alternativo & 4 & 41 & .072 & $.069-.074$ & .102 & .823 & .812 & 3.86 \\
Original $^{\mathrm{B}}$ & 7 & 23 & .046 & $.040-.051$ & .053 & .961 & .952 & 2.72 \\
Alternativo $^{\mathrm{B}}$ & 4 & 24 & .087 & $.083-.092$ & .1 & .842 & .823 & 5.25 \\
\hline
\end{tabular}

Nota: $\mathrm{B}=$ corresponde al modelo original y alternativo con menor número de ítems, RMSEA = error cuadrático medio de aproximación, SRMR = residual cuadrático estandarizado, CFI = índice de ajuste comparativo, TLI = índice Tucker-Lewis, $\chi^{2} / \mathrm{gl}=$ razón de Chi-cuadrado sobre los grados de libertad, AIC = criterio de información de Akaike. 
Tabla 2

Soluciones estandarizadas del análisis factorial confirmatorio para el modelo de 7 factores con 23 ítems

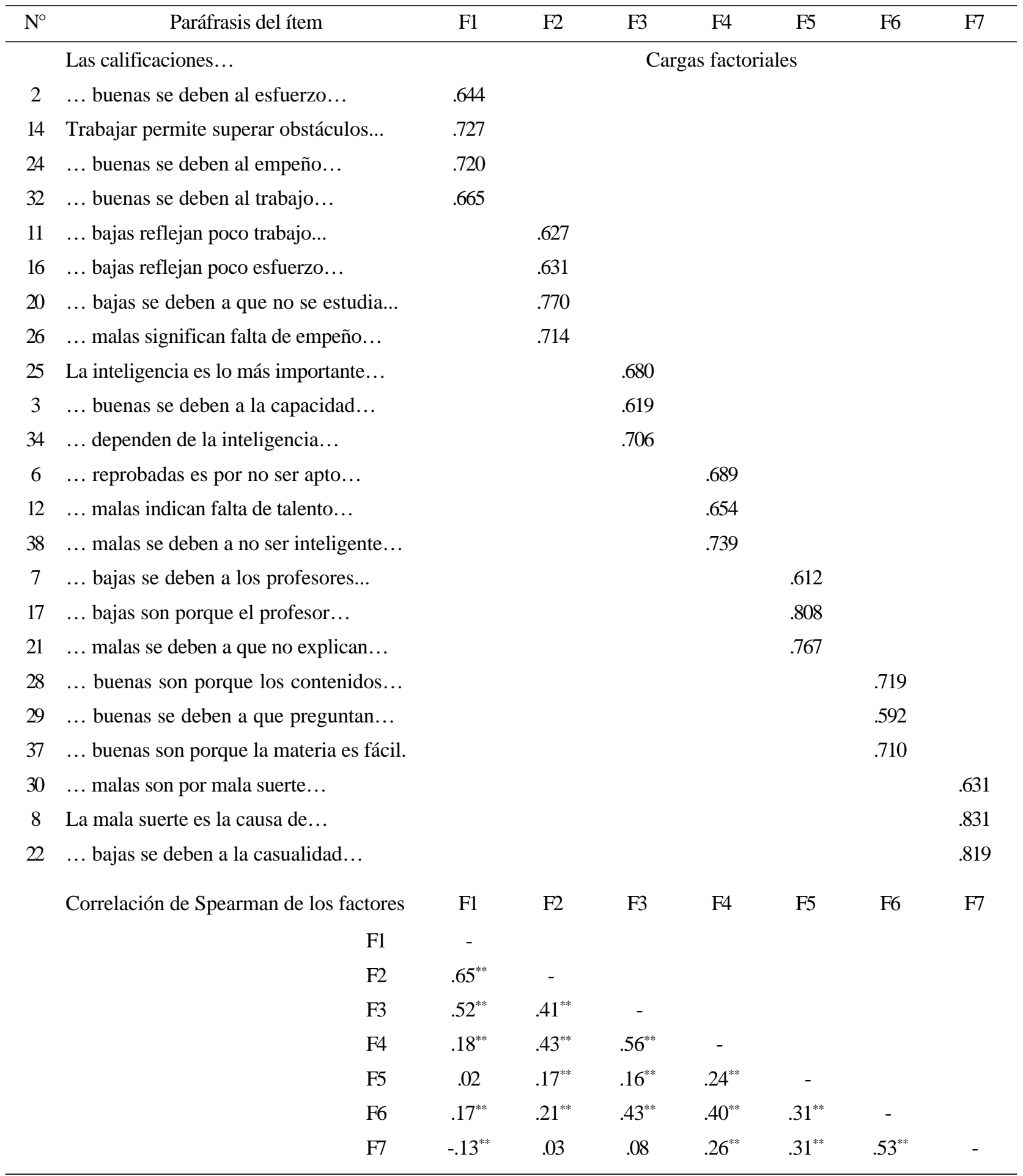

Nota: La correlación es significante al nivel $.05^{*}, .001^{* *}$ (bilateral). F1 = atribución del éxito al esfuerzo, F2 = atribución del fracaso al esfuerzo, F3 = atribución del éxito a la habilidad, F4= atribución del fracaso a la habilidad, F5 = atribución del fracaso al profesor, F6 = atribución del éxito a la suerte, F7 = atribución del fracaso a la suerte, $\mathrm{N}^{\circ}=$ indica la posición del ítem en el cuadernillo. 
Tabla 3

Correlación de Spearman de los factores de atribución del EAT-A con los factores de la escala de motivación del PALS-PI

\begin{tabular}{|c|c|c|c|}
\hline Atribución / Motivación & OMD & OMCA & OMCE \\
\hline Éxito por el esfuerzo & $.19 * *$ & $.14^{* *}$ & $.13^{* *}$ \\
\hline Fracaso por el esfuerzo & $.08^{*}$ & $.19 * *$ & $.14^{* *}$ \\
\hline Éxito por la habilidad & $.10^{*}$ & $.18^{* *}$ & $.19 * *$ \\
\hline Fracaso por la habilidad & -.05 & $.19 * *$ & $.20 * *$ \\
\hline Fracaso por el profesor & -.03 & $.13^{* *}$ & $.14^{* *}$ \\
\hline Éxito por la suerte & -.02 & $.18^{* *}$ & $.17^{* *}$ \\
\hline Fracaso por la suerte & $-.17 * *$ & $.15^{* *}$ & $.15^{*}$ \\
\hline
\end{tabular}

Tabla 4

Coeficiente omega de Mcdonald y confiabilidad compuesta para los factores del modelo original con 23 ítems

\begin{tabular}{lccc}
\hline \multicolumn{1}{c}{$*$ Factor } & Ítems & Omega de Mcdonald & Confiabilidad compuesta \\
\hline Éxito por el esfuerzo & 4 & .77 & .73 \\
Fracaso por el esfuerzo & 4 & .77 & .75 \\
Éxito por la habilidad & 3 & .67 & .66 \\
Fracaso por la habilidad & 3 & .65 & .66 \\
Fracaso por el profesor & 3 & .73 & .72 \\
Éxito por la suerte & 3 & .66 & .66 \\
Fracaso por la suerte & 3 & .68 & .68 \\
\hline
\end{tabular}

Nota: *Atribuciones de las calificaciones académicas. 


\section{Discusión}

Se ha destacado la importancia que tienen las atribuciones causales en los resultados académicos de los estudiantes (Houston, 2016; Zubeldia et al., 2018; Sáez et al., 2018). Sin embargo, los instrumentos que se emplean para examinar las atribuciones en este ámbito suelen carecer o no exhiben evidencias de las propiedades psicométricas que brinden soporte a su estructura factorial y corroboren la validez convergente y confiabilidad en la población objetivo.

Dado lo anterior, el propósito del estudio fue examinar estas propiedades de uno de los instrumentos que frecuentemente se ha empleado en estudiantes hispanohablantes para examinar las atribuciones que hacen de sus calificaciones, el EAT-A.

Los resultados obtenidos apoyan la estructura factorial del EAT-A con siete factores: 1) atribución del éxito al esfuerzo, 2) atribución del fracaso a la falta de esfuerzo, 3) atribución del éxito a la habilidad, 4) atribución del fracaso a la falta de habilidad, 5) atribución del fracaso al profesor, 6) atribución del éxito a la suerte y 7) atribución del fracaso a la suerte. En esta solución factorial es posible identificar las dimensiones de locus, estabilidad y controlabilidad (Graham, 2020; Weiner, 2018) en las causas por las que los estudiantes atribuyen sus calificaciones. De acuerdo con estos autores, la dimensión de locus quedaría reflejada con el esfuerzo y la habilidad como causas internas del estudiante, y la suerte y el profesor como atribuciones externas. La dimensión de estabilidad permite diferenciar el esfuerzo y la suerte como atribuciones inestables, y la habilidad y el profesor como causas estables. La controlabilidad se representaría con el esfuerzo como una causa que el estudiante puede controlar, con los profesores como algo que la institución puede controlar, y con la suerte y la habilidad como causas que no se pueden controlar.

Asimismo, la estructura con siete factores apoya la propuesta teórica de que se recurren a distintas atribuciones para explicar el éxito y el fracaso. Al considerar que los estudiantes se enfrentan a situaciones de éxito y fracaso no sería posible atribuir a una causa estable para ambos resultados. Si el fracaso de un evento se atribuye a causas inestables e incontrolables, entonces se puede esperar un resultado similar en el futuro. En contraste, cuando se consideran controlables y estables, aumenta la posibilidad de obtener un resultado diferente (Haynes et al., 2009). Por tanto, resulta conveniente que el EAT-A permita distinguir los tipos de resultados para identificar las atribuciones que los estudiantes hacen del éxito y del fracaso.

$\mathrm{Al}$ respecto, se ha observado que los resultados favorables y adversos tienden a atribuirse a diferentes tipos de causas, los éxitos a la propia capacidad o al esfuerzo, y los fracasos a causas externas a uno mismo (Matalinares et al., 2010; Muwonge \& Ssenyonga1, 2015), fenómeno que podría explicarse por la tendencia a preservar el autoconcepto y la autoestima (Alonso \& Sánchez, 1992; Caso \& Hernández, 2010).

En cuanto a la validez convergente, en general los resultados muestran que los factores del EAT-A presentan mayor asociación entre sí que con los factores de las metas de logro. Asimismo, es posible destacar asociaciones que se han observado en la literatura entre los tipos de atribuciones y las metas de motivación. Se ha planteado que los estudiantes se encuentran más motivados para aprender cuando consideran que los éxitos se deben a causas internas y controlables. Contrariamente, cuando los fracasos son vistos como incontrolables o estables, existe una menor motivación para alcanzar el objetivo (Lagos et al., 2016; Hamm et al., 2017; Haynes et al., 2009; Pekrun \& Perry, 2014; Weiner, 2018).

En este estudio, las metas de dominio muestran asociación negativa con las atribuciones de éxito y fracaso externas, así como con la atribución interna de fracaso por la habilidad. Lo anterior refleja que el estudiante con motivación por aprender se responsabiliza de sus resultados académicos debido a que los atribuye a causas internas y controlables, como el esfuerzo. Al respecto, se ha informado que el 
estudiante que considera que las causas de su éxito radican en sí mismo y que son controlables, refiere mayor motivación orientada al dominio que cuando considera que las causas son externas, estables e incontrolables (Haynes et al., 2009; Matalinares et al., 2010; Miñano \& Castejón, 2011). En contraste, las metas de competencia de acercamiento y evitación asocian más los éxitos con la suerte, lo cual, al ser una causa externa, inestable e incontrolable, como la suerte, deslinda de alguna manera al estudiante de la responsabilidad de sus resultados. Asimismo, es posible observar que este tipo de metas presentan mayor asociación con la atribución de los éxitos y los fracasos a la habilidad que las metas de dominio. De acuerdo con la motivación de logro, esto podría implicar que los estudiantes con metas de competencia tienen la noción de que las calificaciones son un reflejo de sus habilidades y por ello buscan la calificación, para demostrarse «buenos» ante los demás, más que por aprender (Elliot, 1999; Regueiro et al., 2018).

Por último, los siete factores de la versión con 23 ítems del EAT-A presentan una consistencia interna adecuada (Ventura-León \& Caycho-Rodríguez, 2017). La presencia de ítems que comprometían el ajuste de ambos modelos es un aspecto relevante de los análisis realizados. Al igual que en el estudio de Jurado et al. (2016), fue necesario eliminar ítems que no discriminaban con sesgo o que presentaban menor carga factorial en cada uno de los factores. La razón de que algunos ítems no funcionen, probablemente, se deba a que el contenido de estos ítems no corresponde con la forma de pensar de los estudiantes en la actualidad o en la localidad que se considera en este estudio, o bien a que no se realizó el procedimiento estadístico adecuado para integrar los factores originales. De lo anterior, se desprende la inminente necesidad de examinar la validez de constructo de los instrumentos de medición antes de hacer inferencias de los resultados.

Una aportación importante de esta investigación es el uso del análisis factorial confirmatorio para brindar evidencia de validez de constructo al EAT-
A, debido a que previamente se había analizado su validez únicamente mediante el análisis de componentes principales (Alonso, 1987). Esta evidencia corrobora que los factores del instrumento se pueden categorizar de acuerdo con las dimensiones atribucionales (Graham, 2020; Weiner, 2018) y el tipo de resultado (Matalinares et al., 2010; Muwonge \& Ssenyonga1, 2015) que se han planteado en la literatura. La evidencia de las propiedades psicométricas del EAT-A exhibe a este instrumento como una opción actualizada para seguir construyendo el campo de conocimiento concerniente a la importancia y a los alcances que tienen las atribuciones en los resultados académicos. Asimismo, la asociación de las atribuciones con la motivación que refieren los estudiantes brinda evidencia de la importancia de contemplar ambas variables para favorecer los resultados académicos.

Por otro lado, es necesario mencionar que el presente estudio tuvo limitaciones que podrían solventarse en estudios futuros. En primer lugar, sería ideal disponer de un muestreo probabilístico que favoreciera la representatividad de los resultados y contemplar al menos otra muestra para analizar si el instrumento señala invarianza de la solución factorial. Asimismo, podrían contemplarse muestras de otros países hispanohablantes para establecer si existe invarianza con otros países del mundo hispano o si, por el contrario, se presentan diferencias transculturales. En segundo lugar, resultaría conveniente indagar de qué manera los estudiantes conciben el esfuerzo, la habilidad, el profesor y la suerte de acuerdo con las dimensiones de locus, estabilidad y controlabilidad; ya que se ha observado que pueden tener distinto significado para estos (Gonzalez, 2016). La forma de categorizar las causas en sus dimensiones podría hablar de distintos tipos de estudiantes. Por ejemplo, las habilidades pueden plantearse como algo estable o una característica inestable que se desarrolla con la práctica. De acuerdo con Dweck y Yeager (2019), promover en los estudiantes que las habilidades son inestables y que se incrementan con la práctica favorece sus 
resultados académicos, así como su motivación por aprender.

\section{Conclusiones}

En este estudio, el instrumento EAT-A se perfila como un instrumento de medida breve y de fácil aplicación que dispone de evidencias de validez y estimación de confiabilidad para examinar las atribuciones que los estudiantes hacen de sus calificaciones. Dado que las calificaciones son uno de los resultados que mayor impacto tienen en la trayectoria académica de los estudiantes (Brookhart et al., 2016), resulta imprescindible examinar a qué factores las atribuyen los estudiantes: a su habilidad, a su esfuerzo o a algún factor externo, como los profesores o la suerte. Distinguir las atribuciones que refieren los estudiantes de sus calificaciones permitirá explorar de qué manera se asocian con los resultados académicos y con otras variables que inciden en su desempeño. Disponer de esta información podrá orientar la implementación de prácticas preventivas en el aula que promuevan las atribuciones adaptativas, así como dirigir intervenciones orientadas a modificar las atribuciones en los estudiantes que podrían comprometer su formación académica (Hamm et al., 2017; Morris, 2013; Sukariyah \& Assaad, 2015).

\section{Conflicto de intereses}

Por medio del presente se declara que los autores del presente artículo no tienen ningún conflicto de intereses con el contenido del mismo.

\section{Responsabilidad ética}

El presente estudio se realizó contemplando las normas y los lineamientos del Código ético propuesto por la Sociedad Mexicana de Psicología (2010). En particular, se atendieron los artículos 47, 48 y 49 concernientes a los protocolos de investigación. Con la anuencia de las autoridades correspondientes, se informó a los estudiantes el propósito del estudio, así como las características de su participación: voluntaria, anónima y autónoma. Cabe aclarar que en esta investigación no se realizaron experimentos con humanos ni animales.

\section{Contribución de autoría}

AMRH: diseño del estudio, recolección de datos, análisis estadístico, redacción, discusión y revisión final del manuscrito.

LHG: diseño del estudio, discusión y revisión final del manuscrito.

\section{Agradecimientos}

Los resultados del presente artículo forman parte de una investigación más amplia que fue llevada a cabo como parte del proyecto doctoral de Alejandro Manuel Rodríguez Huitrón. Se extiende un agradecimiento al Consejo Nacional de Ciencia y Tecnología (Conacyt), el cual le otorgó una beca para cursar el grado (becario 588637). Asimismo, se agradece a la Dirección General de Asuntos del Personal Académico de la UNAM que apoyó la realización del estudio mediante el Proyecto PAPIIT IN304016.

\section{Referencias}

Ahmed, S. F., Tang, S., Waters, N. E., \& Davis-Kean, P. (2019). Executive Function and Academic Achievement: Longitudinal Relations from Early Childhood to Adolescence. Journal of Educational Psychology, 111(3), 446-458. https://doi.org/10.1037/edu0000296

Alavi, M., Visentin, D. C., Thapa, D. K., Hunt, G. E., Watson, R., \& Cleary, M. (2020). Exploratory Factor Analysis and Principal Component Analysis in Clinical Studies: Which One Should You Use? Journal of Advanced Nursing, 76(8), 1886-1889. https://doi.org/10.1111/ jan.14377

Alonso, J. (1987). Estudio sobre la validez de constructo de los cuestionarios MAPE, EAT y ECO. Estudios de Psicología, 8(29-30), 45-69. https://doi.org/10.1080/ 02109395.1987.10821480

Alonso, J., \& Sánchez, J. (1992). Estilos atributivos y motivación: el cuestionario EAT. En J. Alonso (ed.), 
Motivar en la adolescencia: teoría, evaluación e intervención (pp. 93-133). Ediciones de la Universidad Autónoma de Madrid.

Brookhart, S. M., Guskey, T. R., Bowers, A. J., McMillan, J. H., Smith, J. K., Smith, L. F., Stevens, M. T., \& Welsh, M. E. (2016). A Century of Grading Research: Meaning and Value in the Most Common Educational Measure. Review of Educational Research, 86(4), 803-848. https://doi.org/10.3102/0034654316672069

Buuren, S. van, Groothuis-Oudshoorn, K., \& Robitzsch, A. (2019). Package 'mice': Multivariate Imputation by Chained Equations. https://cran.r-project.org/web/ packages/mice/mice.pdf

Campbell, D. T., \& Fiske, D. W. (1959). Convergent and discriminant validation by the multitrait-multimethod matrix. Psychological Bulletin, 56(2), 81-105. https:// doi.org/10.1037/h0046016

Carpi, A. B., Gómez C. I., Guerrero, C. R., \& Palmero, F. C. (2009). Expectativa de logro, atribución y variables fisiológicas. Análisis y modificación de conducta, 35(152), 19-36.

Caso, J., \& Hernández, L. (2010). Modelo explicativo del bajo rendimiento escolar: un estudio con adolescentes mexicanos. Revista Iberoamericana de Evaluación Educativa, 3(2), 145-159. http://www.rinace.net/riee/ numeros/vol3-num2/art8.pdf

Cerrón, J. C. (2016). Comparación de pruebas de normalidad multivariada. Anales Científicos. 77(2), 141-146. http://dx.doi.org/10.21704/ac.v77i2.483

Clark, L. A., \& Watson, D. (2019). Constructing Validity: New Developments in Creating Objective Measuring Instruments. Psychological Assessment, 31(12), 14121427. https://doi.org/10.1037/pas0000626

Deng, L., \& Chan, W. (2017). Testing the Difference Between Reliability Coefficients Alpha and Omega. Educational and Psychological Measurement, 77(2), 185-203. https://doi.org/10.1177/0013164416658325

Dweck, C. S., \& Yeager, D. S. (2019). Mindsets: A View from Two Eras. Perspectives on Psychological Science, 14(3), 481-496. https://doi.org/10.1177/1745691618804166

Elliot, A. J. (1999). Approach and Avoidance Motivation and Achievement Goals. Educational Psychologist, 34(3), 169-189. https://doi.org/10.1207/s15326985ep 3403_3
Erten, I. H., \& Burden, R. L. (2014). The Relationship between Academic Self-Concept, Attributions, and L2 Achievement. System, 42, 391-401. https://doi.org/10.1 016/j.system.2014.01.006

Farrington, C. A., Roderick, M., Allensworth, E., Nagaoka, J., Keyes, T. S., Johnson, D. W., \& Beechum, N. O. (2012). Teaching Adolescents to Become Learners: The Role of Noncognitive Factors in Shaping School Performance-A Critical Literature Review. University of Chicago Consortium on Chicago School Research.

Foulkes, L., \& Blakemore, S. (2018). Studying individual differences in Human Adolescent Brain Development. Nature Neuroscience, 21(3), 315-323. https://doi.org/ 10.1038/s41593-018-0078-4

Gaeta, M. L., Teruel, M., \& Orejudo, S. (2012). Aspectos motivacionales, volitivos y metacognitivos del aprendizaje autorregulado. Electronic Journal of Research in Educational Psychology, 10(26), 73-94.

Gómez, M. G., Mattiello, G., \& Repetto, A. J. (2011). Proceso de creación y validación de un instrumento que evalúa la creatividad de escolares (VAI). Revista Electrónica de Investigación y Docencia (REID), 5, 7-34.

Gonzalez, A. (2016). Attribution Theory: Dimensions of Causality, Stability and Controllability According to Learners. En C. Gkonou, D. Tatzl \& S. Mercer (eds.), New Directions in Language Learning Psychology, (pp. 209-232). Springer. https://doi.org/10.1007/978-3319-23491-5_12

González-Torres, M., \& Tourón, J. (1992). Autoconcepto y rendimiento escolar: sus implicaciones en la motivación y en la autorregulación del aprendizaje. Eunsa.

Graham, S. (2020). An Attributional Theory of Motivation. Contemporary Educational Psychology, 61, 101861. https://doi.org/10.1016/j.cedpsych.2020.101861

Hair, J. F., Black, W. C., Babin, B. J., \& Anderson R. (2014). Multivariate Data Analysis: A Global Perspective. Pearson New International Edition.

Hamm, J. M., Perry, R. P., Chipperfield, J. G., Heckhausen, J., \& Parker, P. C. (2016). A motivation-enhancing treatment to sustain goal engagement during life course transitions. Motivation and Emotion, 40, 814829. https://doi.org/10.1007/s11031-016-9576-4 
Hamm, J. M., Perry, R. P., Chipperfield, J. G., Murayama, K., \& Weiner, B. (2017). Attribution-Based Motivation Treatment Efficacy in an Online Learning Environment for Students Who Differ in Cognitive Elaboration. Motivation and Emotion, 41(5), 600-616. https:// doi.org/10.1007/s11031-017-9632-8

Haynes, T., Perry, R. P., Stupnisky, R. H., \& Daniels, L. (2009). A Review of Attributional Retraining Treatments: Fostering Engagement and Persistence in Vulnerable College Students. En J. Smart (ed.), Higher Education: Handbook of Theory and Research (pp. 227-272). Springer. https://doi.org/10.1007/978-1-4020-9628-0_6

Houston, D. M. (2016). Revisiting the Relationship between Attributional Style and Academic Performance. Journal of applied social psychology, 46(3), 192-200. https://doi.org/10.1111/jasp.12356

Hu, L., \& Bentler, P. M. (1999). Cutoff Criteria for Fit Indexes in Covariance Structure Analysis: Conventional Criteria versus New Alternatives. Structural Equation Modeling: A Multidisciplinary Journal, 6(1), 1-55. https://doi.org/10.1080/10705519909540118

Jaimes, J. E. (2015). Relación entre comprensión lectora y atribuciones causales de éxito y fracaso en alumnos de tercer año de secundaria de colegios públicos y privados de Lima Metropolitana [tesis de doctorado, Pontificia Universidad Católica de Perú]. Repositorio. http://hdl.handle.net/20.500.12404/5798

Jurado, P. J., Blanco, H., Zueck, M. C., \& Peinado, J. E. (2016). Composición factorial del Cuestionario de Estilos Atributivos área de logros académicos en universitarios mexicanos. Formación universitaria, 9(6), 83-92. http://dx.doi.org/10.4067/S0718-50062 016000600008

Kline, R. B. (1998). Principles and Practice of Structural Equation Modeling. The Guilford Press.

Korkmaz, S., Goksuluk, D., \& Zararsiz, G. (2019). Package 'MVN': Multivariate Normality Tests. https:/cran.rproject.org/web/packages/MVN/MVN.pdf

Lagos, N. G., Inglés, C. J., Ossa, C. J., Gonzálvez-Maciá, C., Vicent-Juan, M., \& García-Fernández, J. M. (2016). Relación entre atribuciones de éxito y fracaso académico y ansiedad escolar en estudiantes chilenos de educación secundaria. Psicología desde el Caribe, 33(2), 146-157. http://dx.doi.org/10.14482/psdc.33.2.7296
Lang, K. M., \& Little, T. D. (2018). Principled Missing Data Treatments. Prevention Science, 19(3), 284-294. https:/ /doi.org/10.1007/s11121-016-0644-5

Lohbeck, A., Grube, D., \& Moschner, B. (2017). Academic Self-Concept and Causal Attributions for Success and Failure Amongst Elementary School Children. International Journal of Early Years Education, 25(2), 190-203. https://doi.org/10.1080/09669760.2017.1301806

Mahmoodi, M. H., \& Doosti, M. (2018). Investigating the Effects of Attributional Retraining Procedures on High School Students' Foreign Language Causal Attributions and their Foreign Language Achievement. The Journal of Language Learning and Teaching, 8(2), 28-44. https:/ /dergipark.org.tr/en/pub/jltl/issue/42266/508509

Martínez-Abad, F., \& Rodríguez-Conde, M. J. (2017). Comportamiento de las correlaciones productomomento y tetracórica-policórica en escalas ordinales: un estudio de simulación. RELIEVE. Revista Electrónica de Investigación y Evaluación Educativa, 23(2), 1-21. https://doi.org/10.7203/relieve.23.2.9476

Matalinares, M., Yaringaño, J., Sotelo, L., Sotelo, N., Arenas, C., Díaz, G., Dioses, G., Encalada, M., Tipacti, R., Bázan, M., \& Huari, Y. (2010). Relación entre los estilos atribucionales y los estilos de aprendizaje en estudiantes de secundaria de Lima Metropolitana. Revista de Investigación en Psicología, 13(2), 101-116. https://doi.org/10.15381/rinvp.v13i2.3716

Matalinares, M., Tueros, R., \& Yaringaño, J. (2009). Adaptación psicométrica del cuestionario de estilos atributivos. Revista de Investigación en Psicología, 12(1), 173-189. https://doi.org/10.15381/rinvp.v12i1.3789

McMillan, W. (2015). Identity and Attribution as Lenses to Understand the Relationship between Transition to University and Initial Academic Performance. African Journal of Health Professions Education, 7(1), 32-38. http://dx.doi.org/10.7196/ AJHPE.356

Midgley, C., Maehr, M. L., Hruda, L. Z., Anderman, E., Anderman, L., Freeman, K., Gheen, M., Kaplan, A., Kumar, R., Middleton, M., Nelson. J., Roeser, R., \& Urdan, T. (2000). Manual for the patterns of adaptive learning scales. University of Michigan. https://doi.org/ 10.1037/t19870-000

Miñano, P., \& Castejón, J. L. (2011). Variables cognitivas y motivacionales en el rendimiento académico en 
Lengua y Matemáticas: un modelo estructural. Revista de Psicodidáctica, 16(2), 203-230. https://www.redalyc. org/articulo.oa?id=17518828003

Monroe, S. (2018). Contributions to Estimation of Polychoric Correlations. Multivariate Behavioral Research, 53(2), 247-266. https://doi.org/10.1080/002731 71.2017 .1419851

Montero, I., \& León, O. (2007). A Guide for Naming Research Studies in Psychology. International Journal of Clinical and Health Psychology, 7(3), 847862. http://www.redalyc.org/articulo.oa?id=33770318

Moraga, F. A. (2017). Metas académicas, estilos atributivos $y$ estrategias de aprendizaje en estudiantes secundarios de establecimientos vulnerables de la Región del BioBío. [tesis de maestría, Universidad de Concepción]. Repositorio. http://repositorio.udec.cl/ jspui/handle/11594/2620

Morris, M. (2013). A Naturalistic Investigation into the Effectiveness of an Attributional Retraining Program for Academic Performance. Social Sciences Directory, 2(2), 16-3. https://www.researchgate.net/publication/ 260555277_A_naturalistic_investigation_into_ the_effectiveness_of_an_attributional_retraining_prog ramme_for_academic_performance

Muñiz, J., Elosua, P., \& Hambleton, R. K. (2013). Directrices para la traducción y adaptación de los tests: segunda edición. Psicothema, 25(2),151-157. http://dx.doi.org/ 10.7334/psicothema2013.24

Muwonge, C. M., \& Ssenyonga, J. (2015). Academic Performance, Causal Attributions, and Hope to Complete Studies among University Students. Journal of Education, Psychology, and Social Sciences, 3, 50-56.

Orcan, F. (2018). Exploratory and Confirmatory Factor Analysis: Which One to Use First. Journal of Measurement and Evaluation in Education and Psychology, 9(4), 414-421. https://doi.org/10.21031/ epod.394323

Otzen, T., \& Manterola, C. (2017). Técnicas de muestreo sobre una población a estudio. International Journal of Morphology, 35(1), 227-232. http://dx.doi.org/ 10.4067/S0717-95022017000100037

Pekrun, R., \& Perry, R. P. (2014). Control-Value Theory of Achievement Emotions. En R. Pekrun \& L. Linnenbrink-
Garcia (eds.), International handbook of emotions in education (pp. 120-141). Routledge.

Peterson, R., \& Kim, Y. (2013). On the Relationship between Coefficient Alpha and Composite Reliability. Journal of Applied Psychology, 98(1), 194-198. https://doi.org/ 10.1037/a0030767

Redondo, J., Ingles, C., \& García-Fernández, J. M. (2014). Conducta prosocial y autoatribuciones académicas en Educación Secundaria Obligatoria. Anales de Psicología, 30(2), 482-489. https://doi.org/10.6018/ analesps.30.2.148331

Regueiro, B., Núñez, J. C., Valle, A., Piñeiro, I., Rodríguez, S., \& Rosário, P. (2018). Motivational Profiles in High School Students: Differences in Behavioural and Emotional Homework Engagement and Academic Achievement. International Journal of Psychology, 53(6), 449-457. https://doi.org/10.1002/ijop.12399

Revelle, W. (2019). Package psych: Procedures for Psychological, Psychometric, and Personality Research. https://cran.rproject.org/web/packages/ psych/psych.pdf

Rikoon, S. H., Liebtag, T., Olivera Aguilar, M., Steinberg, J., \& Robbins, S. (2015). Anticipating College Enrollment: Adapting Success Navigator ${ }^{\circledR}$ for High School Students. ETS Research Report Series, 2, 126. https://onlinelibrary.wiley.com/doi/pdf/10.1002/ ets2.12084

Rosseel, Y., Jorgensen, T., Rockwood, N., Oberski, D., Byrnes, J., Vanbrabant, L., Savalei, V., Merkle, E., Hallquist, M., Rhemtulla, M., Katsikatsou, M., Barendse, M., \& Scharf, F. (2020). Package 'lavaan'. CRAN Repository. https://cran.r-project.org/ web/packages/lavaan/lavaan.pdf

RStudio. (2019). RStudio: Integrated Development for $R$. RStudio. https://www.rstudio.com/products/rstudio

Sáez, F. M., Bustos, C. E., Pérez, M. V., Mella, J. A., Lobos, K. A., \& Díaz, A. E. (2018). Willingness to Study, SelfEfficacy and Causal Attributions in Chilean University Students. Journal of Educational Psychology Propósitos y Representaciones, 6(1), 223-245. http:// dx.doi.org/10.20511/pyr2018.v6n1.179

Sakaki, M., \& Murayama, K. (2013). Automatic Ability Attribution After Failure: A Dual Process View of 
Achievement Attribution. Plos One, 8(5), 1-4. https:// doi.org/10.1371/journal.pone.0063066

Sukariyah, M., \& Assaad, G. (2015). The Effect of Attribution Retraining on the Academic Achievement of High School Students in Mathematics. ProcediaSocial and Behavioral Sciences, 177(1), 345-351. https://doi.org/10.1016/j.sbspro.2015.02.356

UNESCO. (2011). Clasificación internacional normalizada de la educación. http://unesdoc.unesco.org/images/ 0022/002207/220782s.pdf

Ventura-León, J. L., \& Caycho-Rodríguez, T. (2017). El coeficiente Omega: un método alternativo para la estimación de la confiabilidad. Revista Latinoamericana de Ciencias Sociales, Niñez y Juventud, 15(1), 625-627. https://www.redalyc.org/pdf/773/77349627039.pdf

Weiner, B. (2018). The Legacy of an Attribution Approach to Motivation and Emotion: A No-Crisis Zone. Motivation Science, 4(1), 4-14. https://doi.org/10.1037/ mot0000082

Zubeldia, M. E., Díaz, M., \& Goñi, E. (2018). Autoconcepto, atribuciones causales y ansiedad-rasgo del alumnado de conservatorio. Diferencias asociadas a la edad y al género. Psychology, Society \& Education, 10(1), 79102. http://dx.doi.org/10.25115/psye.v10i1.1048

\begin{abstract}
Alejandro Manuel Rodríguez Huitrón
Laboratorio de Investigación en Psicopatología Infanto-Juvenil, Facultad de Psicología, Universidad Nacional Autónoma de México.

Licenciado en Psicología y profesor de asignatura. Su trabajo se centra en la evaluación educativa y en las variables psicológicas que influyen en el rendimiento académico.
\end{abstract}

ORCID: https://orcid.org/0000-0003-0684-2885

Autor corresponsal: alejandro8mrh@gmail.com, alejandro8mrh@comunidad.unam.mx

Laura Hernández-Guzmán

Laboratorio de Investigación en Psicopatología Infanto-Juvenil, Facultad de Psicología, Universidad Nacional Autónoma de México. Ph. D. en Psicología y catedrática de la universidad. Su trabajo se centra en la medición, tratamiento e impacto de las psicopatologías infantil y juvenil.

ORCID: https://orcid.org/0000-0003-3996-9540

lahegu@me.com, lher@unam.mx 